\title{
ABSOLUTE CONTINUITY CHARACTERIZATION SETS
}

\author{
WILLIAM D. L. APPLING
}

\begin{abstract}
Suppose $U$ is a set, $\mathbf{F}$ is a field of subsets of $U, \mathfrak{p}_{A B}$ is the set of all real-valued, bounded finitely additive functions on $\mathbf{F}$, and for each $\rho$ in $\mathfrak{p}_{A B}, \mathbb{Q}_{\rho}$ is the set of all elements of $\mathfrak{p}_{A B}$ absolutely continuous with respect to $\rho, \mathfrak{p}_{A}^{+}$is the set of all nonnegative-valued elements of $\mathfrak{p}_{A B}$, and $\mathfrak{p}_{B}$ is the set of all functions from $\mathbf{F}$ into $\exp (\mathbf{R})$ with bounded range union.

An extension of a previous absolute continuity characterization theorem of the author (Proc. Amer. Math. Soc. 18 (1967), 94-99) is given in the form of a characterization of those subsets $S$ of $\mathfrak{p}_{A B}$ having the property that if each of $\xi$ and $\mu$ is in $\mathfrak{p}_{A}^{+}$, then $\xi$ is in $Q_{\mu}$ iff it is true that if $\alpha$ is in $\mathfrak{p}_{B}$, $\int_{U} \alpha(I) \mu(I)$ and $\int_{U} \alpha(I) \xi(I)$ exist and the function $\int \alpha \mu$ is in $S$, then $\int \alpha \xi$ is in $S$.
\end{abstract}

1. Introduction.Suppose $U$ is a set, $\mathbf{F}$ is a field of subsets of $U, \mathfrak{p}_{A B}$ is the set of all real-valued, bounded finitely additive functions on $\mathbf{F}$, and $\mathfrak{p}_{A}^{+}$is the set of all nonnegative-valued elements of $\mathfrak{p}_{A B}$.

It is the purpose of this paper to give a characterization of certain subsets of $\mathfrak{p}_{A B}$, which, by means of certain integral (see \$2) function inclusion properties, characterize the absolute continuity of an element of $p_{A}^{+}$with respect to an element of $\mathfrak{p}_{A}^{+}$. It will be seen that this description is a generalization of an easy consequence (see Theorem 1.1 below) of a previous theorem of the author [2] which we now state. For each $\rho$ in $\mathfrak{p}_{A B}$, let $Q_{\rho}$ denote the set of all elements of $\mathfrak{p}_{A B}$ absolutely continuous with respect to $\rho$.

THEOREM 1.A.1 [2]. If each of $\mu$ and $\xi$ is in $\mathrm{p}_{A}^{+}$, then the following two statements are equivalent:

(1) If $\alpha$ is a bounded function from $\mathbf{F}$ into the nonnegative numbers such that each of the integrals (see $\$ 2) \int_{U} \alpha(I) \mu(I)$ and $\int_{U} \alpha(I) \xi(I)$ exists and $\int_{U} \alpha(I) \mu(I)$ $=0$, then $\int_{U} \alpha(I) \xi(I)=0$, and

(2) $\xi$ is in $\mathbb{Q}_{\mu}$.

Now, easy extensions of the methods used in [2] to prove Theorem 1.A.1, together with elementary considerations involving differential equivalence (see \$2) yield the following theorem, whose argument we leave to the reader. Let $\mathfrak{p}_{B}$ denote the set of all functions from $\mathbf{F}$ into $\exp (\mathbf{R})$ with bounded range union.

Presented to the Society, June 24, 1974; received by the editors November 13, 1974.

AMS (MOS) subject classifications (1970). Primary 28A25; Secondary 28A10.

Key words and phrases. Set function with bounded range union, bounded finitely additive set function, set function integral (variational integral), absolute continuity characterization set. 
THEOREM 1.1. If each of $\mu$ and $\xi$ is in $\mathfrak{p}_{A}^{+}$, then the following two statements are equivalent:

(1) If $\alpha$ is in $\mathfrak{p}_{B}$ and each of the integrals $\int_{U} \alpha(I) \mu(I)$ and $\int_{U} \alpha(I) \xi(I)$ exists and the function (see $\$ 2) \int \alpha \mu$ is $(\theta)$, the zero function, then $\int \alpha \xi=\theta$, and

(2) $\xi$ is in $\mathbb{Q}_{\mu}$.

If, in the statement of the above theorem, we substitute the statement " $\int \alpha \mu$ is in $\{\theta\}$ " for " $\int \alpha \mu$ is $\theta$ ", and " $\int \alpha \xi$ is in $\{\theta\}$ " for " $\int \alpha \xi=\theta$ ", we see that $\{\theta\}$ is an absolute continuity characterization set in accordance with the following

Definition. The statement that $S$ is an absolute continuity characterization set means $S \subseteq \mathfrak{p}_{A B}$, and if each of $\mu$ and $\xi$ is in $\mathfrak{p}_{A}^{+}$, then $\xi$ is in $\mathbb{Q}_{\mu}$ iff it is true that if $\alpha$ is in $\mathfrak{p}_{B}$ and each of the integrals $\int_{U} \alpha(I) \mu(I)$ and $\int_{U} \alpha(I) \xi(I)$ exists and $\int \alpha \mu$ is in $S$, then $\int \alpha \xi$ is in $S$.

Now, we shall let, for each $\rho$ in $\mathfrak{p}_{A B}, 9(\rho)$ denote the set all $\alpha$ in $\mathfrak{p}_{B}$ such that $\int_{U} \alpha(I) \rho(I)$ exists.

In this paper we give a characterization of the absolute continuity characterization sets in the form of the following extension of Theorem 1.1 (see $\S 3$ ):

THEOREM 3.1. If $S \subseteq \mathfrak{p}_{A B}$, then the following two statements are equivalent:

(1) $S$ is an absolute continuity characterization set, and

(2) the following two statements hold:

(i) if $\kappa$ is in $S$ and $\rho$ is in $\mathbb{Q}_{\kappa}$, then $\int \tau(\kappa)|\rho|$ is in $S$, where $\tau(\kappa)$ is the function from $\mathbf{F}$ into $\exp (\mathbf{R})$ given by

$$
\tau(\kappa)(I)=\left\{\begin{array}{l}
\{1\} \text { if } 0 \leq \kappa(I), \\
\{-1\} \text { if } \kappa(I)<0
\end{array}\right.
$$

and

(ii) if $\zeta$ is in and $S \cap \mathfrak{p}_{A}^{+}$and $\zeta(U)>0$, then there is $\chi$ in $9(\zeta)$ such that $\int \chi \zeta$ is not in $S$.

2. Preliminary theorems and definitions. We refer the reader to [4] for the notions of subdivision, refinement, integral and integral function that we shall use in this paper. The reader is also referred to [4] for a statement of Kolmogoroff's 5 ] differential equivalence theorem and certain of its implications about the existence and equivalence of various integrals that we shall consider.

In subsequent paragraphs, when the existence of an integral or the equivalence of an integral to an integral is an easy consequence of matters of this section preceding whatever discussion is at hand, the integral need only be written or the equivalence assertion made, and the proof left to the reader.

We now state three versions of previous theorems of the author, appropriately modified for the setting of this paper. The arguments for the theorems as stated below carry over from the originals with only minor modifications, and we therefore state them without proof. In each case the reference indicated refers, of course, to the paper in which the original version of the theorem appears.

THEOREM 2.A.1 [1]. If $\xi$ is in $\mathfrak{p}_{A B}$, then $9(\xi)=9\left(\int|\xi|\right)$. 
Theorem 2.A.2 [2]. If $\mu$ is in $\mathfrak{p}_{A}^{+}$and $\xi$ is in $\mathbb{Q}_{\mu} \cap \mathfrak{p}_{A}^{+}$, then $\mathscr{q}(\mu) \subseteq \mathscr{G}(\xi)$.

THEOREM 2.A.3 [3]. If $\mu$ is in $p_{A}^{+}, \xi$ is in $\mathbb{Q}_{\mu} \cap p_{A}^{+}$, and $\alpha$ is an element of $\Phi(\mu)$ with range union a subset of the nonnegative numbers, then $\int \alpha \xi$ is absolutely continuous with respect to $\int \alpha \mu$.

We see, from elementary considerations of differential equivalence, that the statement of Theorem 2.A.3 remains true if the nonnegativeness condition on $\alpha$ is removed; accordingly we shall refer to this strengthened version of Theorem 2.A.3 as Theorem 2.A.4.

We end this section with a theorem that we shall use in proving Theorem 3.1. We state two definitions.

Definition. If $\alpha$ is a function from $\mathbf{F}$ into $\exp (\mathbf{R})$, then $\chi(\alpha)$ is the function with domain $\mathbf{F}$ such that if $I$ is in $\mathbf{F}$, then $\chi(\alpha)(I) \subseteq\{-1,1\}$ and contains -1 iff for some $y$ in $\alpha(I), y<0$, and contains 1 iff for some $y$ in $\alpha(I), 0 \leq y$.

Definition. If $\xi$ is in $\mathfrak{p}_{A B}$, then $\tau(\xi)$ is the function with domain $\mathbf{F}$ such that if $I$ is in $\mathbf{F}$, then $\tau(\xi)(I)=\{1\}$ if $0 \leq \xi(I)$, and $\tau(\xi)(I)=\{-1\}$ if $\xi(I)<0$.

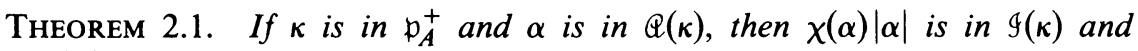
$\int \chi(\alpha)|\alpha| \kappa=\int \alpha \kappa$.

Proof. Suppose $V$ is in $\mathbf{F}$ and $0<c$. Differential equivalence implies that there is a subdivision $\mathscr{D}$ of $V$ such that if $\mathbb{E}$ is a refinement of $\mathscr{D}$ and for each $I$ in \&, $v(I)$ is in $\alpha(I)$, then

$$
\sum_{\kappa}\left|v(I) \kappa(I)-\int_{I} \alpha(J) \kappa(J)\right|<\frac{c}{4} .
$$

Now, suppose \& is a refinement of $\mathscr{D}$ and for each $I$ in $\mathbb{E}, a(I)$ is in $\alpha(I)$, and $d(I)$ is in $\chi(\alpha)(I)$. For each $I$ in \& there is $a^{*}(I)$ in $\alpha(I)$ such that $d(I)\left|a^{*}(I)\right|=a^{*}(I)$. Thus

$$
\begin{aligned}
\mid \int_{V} \alpha(J) \kappa(J) & -\sum_{\mathscr{F}} d(I)[|a(I)| \kappa(I)] \mid \\
\leq & \sum_{\mathscr{E}}\left|\int_{I} \alpha(J) \kappa(J)-a^{*}(I) \kappa(I)\right| \\
& +\sum_{\mathscr{E}}\left|\left[d(I)\left|a^{*}(I)\right|\right]-d(I)\right| a(I) \| \kappa(I) \\
< & \frac{c}{4}+\sum_{\mathscr{E}}|d(I)||| a^{*}(I)|-| a(I)|| \kappa(I) \\
\leq & \frac{c}{4}+\sum_{\mathscr{E}}\left|a^{*}(I)-a(I)\right| \kappa(I) \\
\leq & \frac{c}{4}+\sum_{\mathscr{E}}\left|a^{*}(I) \kappa(I)-\int_{I} \alpha(J) \kappa(J)\right| \\
& +\sum_{\mathscr{F}}\left|\int_{I} \alpha(J) \kappa(J)-a(I) \kappa(I)\right| \\
< & c / 4+c / 4+c / 4<c .
\end{aligned}
$$

Therefore $\chi(\alpha)|\alpha|$ is in $I(\kappa)$ and $\int \chi(\alpha)|\alpha| \kappa=\int \alpha \kappa$. 
3. The characterization theorem. In this section we prove Theorem 3.1 , as stated in the introduction.

Proof of Theorem 3.1. Suppose (1) is true. We first show that (2)(i) is true. Suppose $\kappa$ is in $S$ and $\rho$ is in $\mathbb{Q}_{\kappa}$. If $V$ is in $\mathbf{F}$, then

$$
\kappa(V)=\int_{V} \kappa(I)=\int_{V} \tau(\kappa)(I)|\kappa(I)|=\int_{V} \tau(\kappa)(I) \int_{I}|\kappa(J)|,
$$

so that $\tau(\kappa)$ is in $9\left(\int|\kappa|\right)$ and $\int\left[\tau(\kappa) \int|\kappa|\right]$ is in $S$, and since, by Theorem 2.A.2, $\tau(\kappa)$ is in $g\left(\int|\rho|\right)$, it follows that $\int \tau(\kappa)|\rho|$ is in $S$. Therefore (2)(i) is true.

We now show that (2)(ii) is true. We first note that (2)(i) implies that $\theta$ is in $S$. Now suppose that $\zeta$ is in $S \cap \mathfrak{p}_{A}^{+}$and $\zeta(U)>0$. Then $\zeta$ is not in $\mathbb{Q}_{\theta}$. Therefore there is some $\nu$ in $\mathscr{G}(\zeta) \cap \mathscr{G}(\theta)$ such that $\int \nu \theta$ is in $S$ (trivially) and $\int \nu \zeta$ is not in $S$. Therefore (2)(ii) is true.

Therefore (1) implies (2).

Now suppose (2) is true.

Suppose first that $\mu$ is in $\mathfrak{p}_{A}^{+}, \xi$ is in $\mathbb{Q}_{\mu} \cap \mathfrak{p}_{A}^{+}, \alpha$ is in $g(\mu) \cap g(\xi)$ and $\int \alpha \mu$ is in $S$. Let $\kappa=\int \alpha \mu$. By Theorem 2. A. $4, \int \alpha \xi$ is in $\mathbb{Q}_{\kappa}$, and since $\kappa$ is in $S$, it follows from (2)(i) that $\int\left[\tau(\kappa) \int|\alpha \xi|\right]$ is in $S$. By Theorem 2.1, we have the following extstence and equalities:

$$
\int \chi(\alpha)|\alpha| \mu=\int \alpha \mu, \quad \int \chi(\alpha)|\alpha| \xi=\int \alpha \xi
$$

Now,

$$
\begin{aligned}
\int_{U} \mid \tau(\kappa) & (I)-\chi(\alpha)(I)|| \alpha(I) \mid \mu(I) \\
= & \int_{U}\left|\int_{I} \tau(\kappa)(J)\right| \alpha(J)\left|\mu(J)-\int_{I} \chi(\alpha)(J)\right| \alpha(J)|\mu(J)| \\
= & \int_{U}\left|\int_{I} \tau(\kappa)(J)\right| \kappa(J)\left|-\int_{I} \alpha(J) \mu(J)\right| \\
= & \int_{U}|\kappa(I)-\kappa(I)|=0 .
\end{aligned}
$$

Therefore, by Theorem 1.1,

$$
\begin{aligned}
0 & =\int_{U}|\tau(\kappa)(I)-\chi(\alpha)(I)||\alpha(I)| \xi(I) \\
& =\int_{U}\left|\int_{I} \tau(\kappa)(J)\right| \alpha(J)\left|\xi(J)-\int_{I} \chi(\alpha)(J)\right| \alpha(J)|\xi(J)| \\
& =\int_{U}\left|\int_{I} \tau(\kappa)(J) \int_{J}\right| \alpha\left(J^{\prime}\right) \xi\left(J^{\prime}\right)\left|-\int_{I} \alpha(J) \xi(J)\right| .
\end{aligned}
$$

Since, as previously stated, $\int\left[\tau(\kappa) \int|\alpha \xi|\right]$ is in $S$, it follows that $\int \alpha \xi$ is in $S$.

Now, suppose, conversely, that each of $\mu$ and $\xi$ is in $\mathfrak{p}_{A}^{+}$, and that if $\alpha$ is in $g(\mu) \cap \mathscr{G}(\xi)$ and $\int \alpha \mu$ is in $S$, then $\int \alpha \xi$ is in $S$. We again note that (2)(i) implies that $\theta$ is in $S$. Suppose that $\beta$ is in $\mathscr{G}(\mu) \cap 9(\xi)$ and $\int \beta \mu=\theta$. Now, since $\theta=\int \beta \mu$, it follows that $\theta=\int|\beta| \mu$, and since therefore $\int|\beta| \mu$ is in $S$, it follows that $\int|\beta| \xi$ is in $S \cap \mathfrak{p}_{A}^{+}$. Suppose $\int \beta \xi \neq \theta$, i.e., $\int_{U}|\beta(I)| \xi(I)>0$. By 
(2)(ii) it follows that there is $\delta$ in $9\left(\int|\beta| \xi\right)$ such that $\int \delta|\beta| \xi$ is not in $S$. It clearly follows that $\delta|\beta|$ is in $9(\xi)$. Now, since $\int|\beta| \mu=\theta$, it follows that if $V$ is in $\mathbf{F}, \mathfrak{D}$ is a subdivision of $V$, and for each $I$ in $\mathfrak{D}, d(I)$ is in $\delta(I)$, then $\sum_{\mathscr{D}} d(I) \int_{I}|\beta(J)| \mu(J)=0$, so that $\delta$ is in $g\left(\int|\beta| \mu\right)$ and $\theta=\int\left(\delta \int|\beta| \mu\right)$ $=\int(\delta|\beta|) \mu$, so that it follows that $\delta|\beta|$ is in $9(\mu) \cap 9(\xi)$ and $\int(\delta|\beta| \mu)$ is in $S$, so that $\int(\delta|\beta|) \xi$ is in $S$, a contradiction. Therefore $\int \beta \xi=\theta$. Therefore, by Theorem $1.1, \xi$ is in $\mathbb{Q}_{\mu}$.

Therefore (2) implies (1).

Therefore (1) and (2) are equivalent.

\section{REFERENCES}

1. W. D. L. Appling, Interval functions and the Hellinger integral, Duke Math. J. 29 (1962), 515-520. MR 25 \#4075.

2. - Some integral characterizations of absolute continuity, Proc. Amer. Math. Soc. 18 (1967), 94-99; Addendum, ibid. 24 (1970), 788-793. MR 34 \#4446; 41 \#406.

3. - Two inclusion theorems for real-valued summable set functions, Rend. Circ. Mat. Palermo (2) 18 (1969), 293-306. MR 45 \# 7007.

4. - Set functions, finite additivity and distribution functions, Ann. Mat. Pura Appl. 96 (1973), 265-287.

5. A. Kolmogoroff, Untersuchen über den Integralbegriff, Math. Ann. 103 (1930), 654-696.

Department of Mathematics, North Texas State University, Denton, Texas 76203 